\title{
Study of the inhibitory ability of inorganic di- and polyphosphate compositions
}

\author{
Aktoty Niyazbekova ${ }^{l}$, Timur Shakirov ${ }^{I^{*}}$, and Mayra Almagambetova ${ }^{1}$ \\ ${ }^{1}$ Zhangir khan West Kazakhstan agrarian-technology University, Higher School of Petroleum, Gas \\ and Chemical Engineering, 090000 Uralsk, Kazakhstan
}

\begin{abstract}
The article considers the anticorrosive properties of inorganic di-and polyphosphate compositions in relation to St-3 steel, depending on the $\mathrm{pH}$ of the medium, the nature and concentration of phosphate, and the nature of the modifier ion. The research was conducted using GOST-based methods: gravimetry, potentiometry, photocolorimetry, infrared spectroscopy and scanning electron microscopy. On the basis of experimental data, quantitative indicators of corrosion process were determined: the rate of corrosion process, the degree of protection, the depth index, the coefficient of inhibition and the assessment of the stability of the formed film on the ball scale of corrosion resistance against steel. The analysis of experimental data allows to establish the influence of the above factors on the corrosion processes in the systems under study. The experimental data are supplemented by thermodynamic calculations of the corrosion process parameters, the results of which correlate well with the kinetic data of the process under study. In the course of the research work, the analysis of corrosion deposits was also carried out. The regularities established during the work contribute to the creation of effective di-and polyphosphate inhibitors with the highest degrees of protection.
\end{abstract}

\section{Introduction}

The problem of corrosion is one of the most important in industry, transport and agriculture. Experts estimate the total annual cost of corrosion control at 3-5 \% of the gross domestic product $[1,2]$. According to research by the international Association of corrosion engineers (NACE), the cost of US corrosion control in 2011, for example, amounted to almost $\$ 1$ trillion. The numbers are not comforting, and encourage large companies around the world to engage in thorough anti-corrosion protection.

Most of the costs are actually the cost of anti-corrosion protection, losses in case of equipment failure due to corrosion, as well as the cost of replacing damaged components and, of course, all sorts of accidents. One of the most common ways to reduce the level of corrosion losses in the operation of industrial equipment is the use of corrosion inhibitors $[3,4]$. To date, considerable practical experience has been accumulated in their use. However, the difference in the corrosive aggressiveness of working environments and

* Corresponding author: shakirov_1985@mail.ru 
changes in the operating conditions of equipment and structures at different stages of development put forward new requirements for the selection of inhibitors and improvement of the inhibitor protection technology [5].

Despite the wide range of reagents available, there is a constant search for new inhibitors and inhibiting compositions that can provide a comprehensive protective effect. In this regard, the relevance of this work is to find and implement complex inhibitors to ensure efficient and reliable operation of industrial equipment.

\section{Research methodology}

The purpose of this work is to study effective corrosion inhibitors of complex action.

The following were studied as inhibitors: sodium dihydrodiphosphate $\mathrm{Na}_{2} \mathrm{H}_{2} \mathrm{P}_{2} \mathrm{O}_{7}$, sodium diphosphate $\mathrm{Na}_{4} \mathrm{P}_{2} \mathrm{O}_{7}$, sodium cyclotriphosphate $\mathrm{Na}_{3} \mathrm{P}_{3} \mathrm{O}_{9}$, sodium cyclohexaphosphate $\mathrm{Na}_{6} \mathrm{P}_{6} \mathrm{O}_{18}$. Trivalent ions of iron, aluminum, and lanthanum were chosen as modifier ions.

The corrosion test procedure was generally accepted $[6,7,8]$. Rectangular steel plates $30 \times 20 \times 3 \mathrm{~mm}$ in size were used. The duration of the experiments is $24-480$ hours. The corrosion rate was estimated by the weight loss of the samples after $24,48,72,96,120,240$ hours.

Quantitative indicators of corrosion processes were calculated according to the formulas, the measurement uncertainty was estimated according to the algorithm, using the Student's coefficient with a confidence probability of $0.95[9,10]$.

In the course of the work, potentiometric determinations of the $\mathrm{pH}$ of corrosive media were carried out using a combined glass electrode and ionomer, the conductometric method, and photocolorimetric determination of the iron (III) content with potassium thiocyanate $[11,12]$. And also analyzes of corrosion deposits were carried out on infra-red specroscopy and scanning electron microscopy.

\section{Results and its discussion}

36 multicomponent systems were constructed in order to conduct research on the inhibitory ability of phosphate compounds.

In diphosphate model systems, the passage of reactions is indicated by changes in the concentration of modifier ions, iron (III) ions, and phosphate ions. This relationship is shown in figure 1 for a system with a lanthanum ion, where the inhibitory effect is most effective.

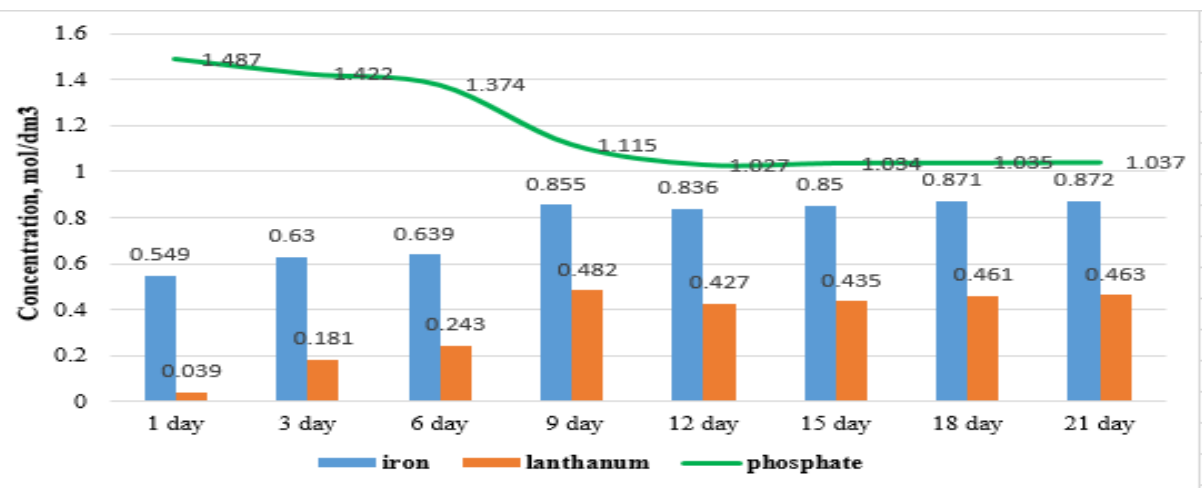

Fig. 1. The dependence of changes in the concentration of the ion of the modifier of lanthanum, iron, phosphate on time for sodium dihydrodiphosphate. 
As shown in the figure, the plate dissolves and the iron passes into the solution under the influence of the medium and the system components. There is an increase in the concentration of iron in the solution in this system on the ninth day. The concentration of phosphate ions at the same time decreases. This indicates that there is a process of interaction between phosphate ions and iron and the formation of corrosive deposits. On the twelfth day, the concentration of iron, lanthanum and phosphorus ions decreases in this system. Apparently, a compound is formed in the system, which inhibits the process of dissolution of iron. Subsequently, the system again shows a slight increase in the concentration of all components of the system. Hence, it can be assumed that the formed compound does not have sufficient strength and degree of protection. The effectiveness of the inhibitory action is only $8.43 \%$. This is the highest degree of protection observed among 18 diphosphate systems.

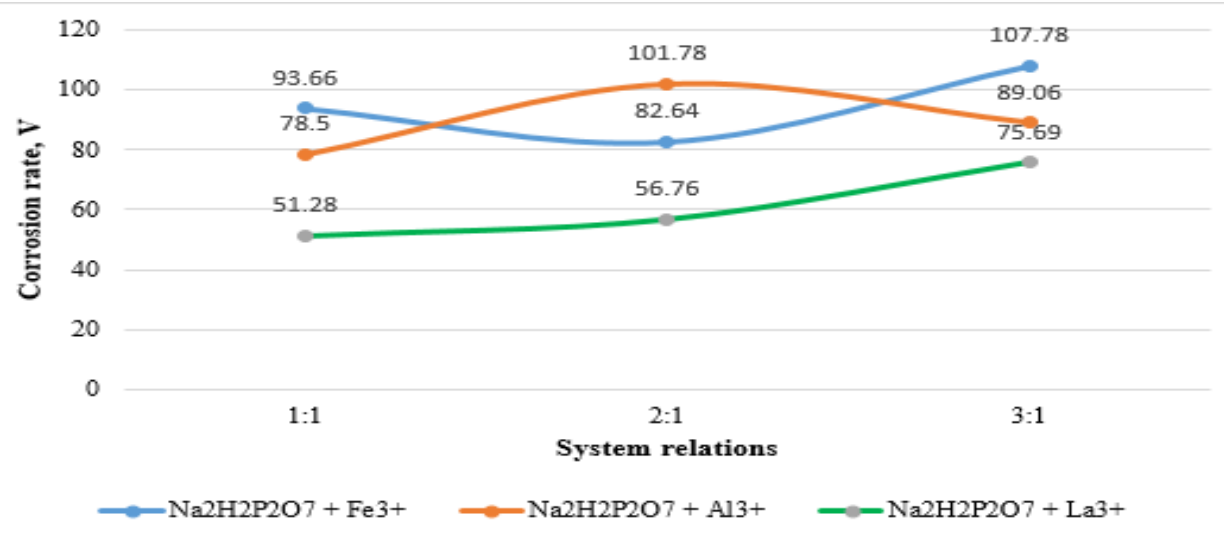

Fig. 2. Dependence of the corrosion rate on the nature of the modifier ion at a different ratio for sodium dihydrodiphosphate.

The change in the corrosion rate and the course of the dependence curve in the presence of modifier ions at different ratios for sodium dihydrodiphosphate is shown in figure 2 . As can be seen from the figure, in the presence of iron ions, when the concentration of phosphate increases, there is a decrease in the corrosion rate at a ratio of $2: 1$, and at a ratio of $3: 1$, it increases back. For a system with an aluminum modifier ion, the inverse relationship is observed. In the system with lanthanum ion there is a gradual increase in the speed of the corrosion process with increasing concentration.

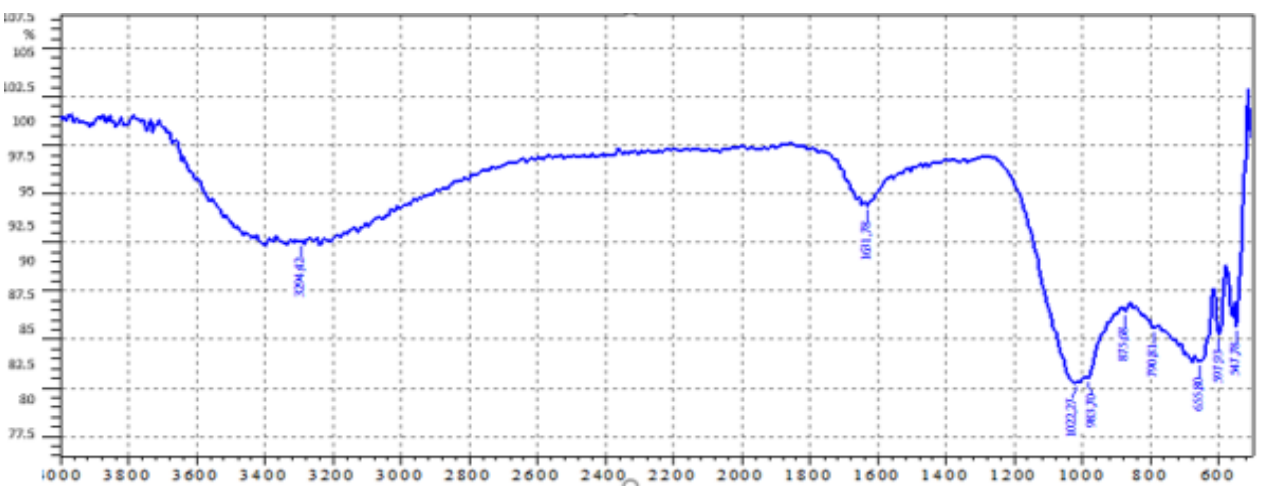

Fig. 3. Spectrogram of the corrosion deposition of the composition of sodium dihydrodiphosphate with lanthanum in a ratio of $1: 1$. 
Figure 3 shows a spectrogram of the corrosion deposition of the sodium dihydrodiphosphate system with an ion modifier in a ratio of $1: 1$. According to the literature $[13,14]$, sodium dihydrodiphosphate is characterized by bands of $1270 \mathrm{~cm}^{-1}$ corresponding to asymmetric $\vee(\mathrm{P}=\mathrm{O})$, and a band of $1092-990 \mathrm{~cm}^{-1}$ to symmetric $\vee(\mathrm{P}=$ $\mathrm{O})$ vibrations. The bands at $884 \mathrm{~cm}^{-1}$ correspond to asymmetric $v$ (P-O-P), and the bands at $792-595 \mathrm{~cm}^{-1}$ correspond to symmetric $v$ (P-O-P) vibrations. The figure shows that in the IR spectrum of the corrosion deposition peaks are formed in the range of $1030-980 \mathrm{~cm}^{-1}$, as well as $1620 \mathrm{~cm}^{-1}$, confirming that another unstable compound is formed.

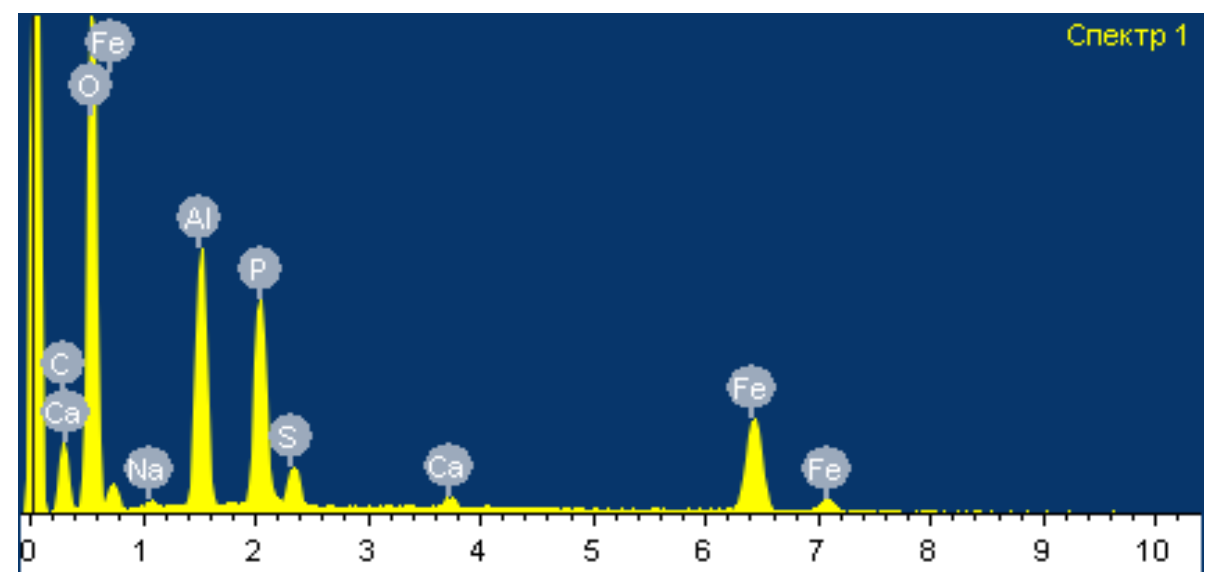

Fig. 4. Spectral scale of elements for corrosion deposition of the composition of sodium dihydrodiphosphate with lanthanum in a ratio of $1: 1$.

Analysis of corrosion deposits using a scanning electron microscope (Figure 4) showed that the corrosion deposits of this system contain oxygen $-46.58 \%$, Fe $-13.36 \%, \mathrm{P}-7.92 \%$. A small amount contains $\mathrm{Na}-0.44 \%$.

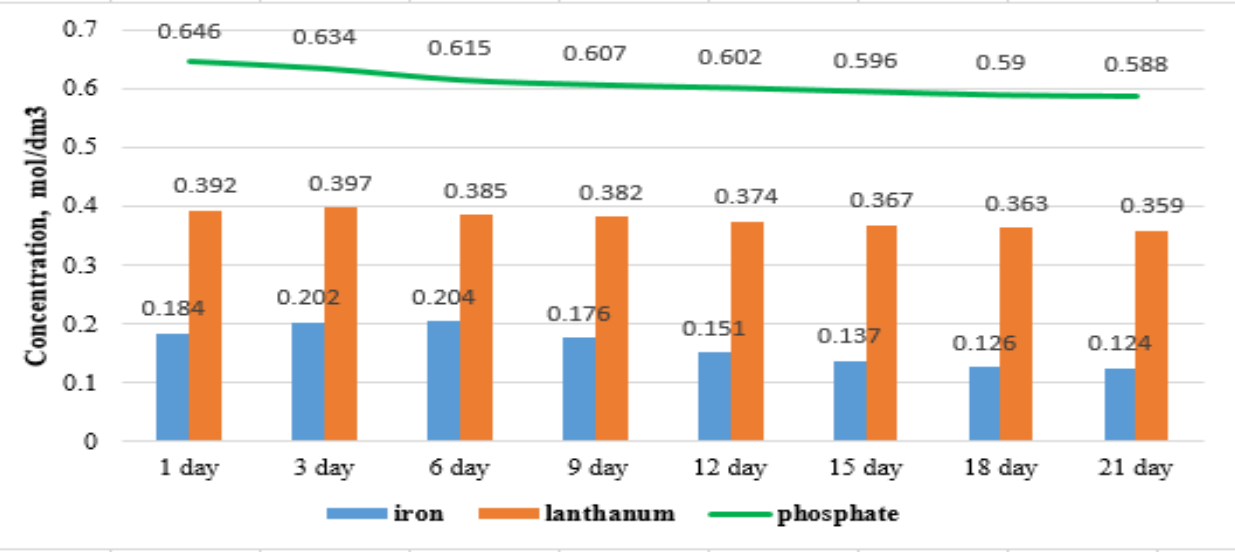

Fig. 5. The dependence of the change in the concentration of the ion of the modifier of lanthanum, iron, phosphate on time for sodium cyclotriphosphate.

Of the eighteen cyclophosphate systems, the sodium cyclotriphosphate system with the lanthanum modifier ion in the ratio $3: 1$ has the greatest inhibitory effect (Figure 5). The effectiveness of the inhibitory effect is $45.86 \%$. From the above dependence it is seen that at the beginning of the experiment, a gradual increase in the concentration of iron and a decrease in the concentration of lanthanum and phosphate ions are observed. On the ninth 
day and thereafter, a gradual decrease in iron concentration is observed. At the same time, the concentration of lanthanum and phosphate ions decreases. This, possibly, indicates that a complex compound is formed, which reduces the transition of iron ions from the steel plate to the solution, and therefore reduces the corrosion process.

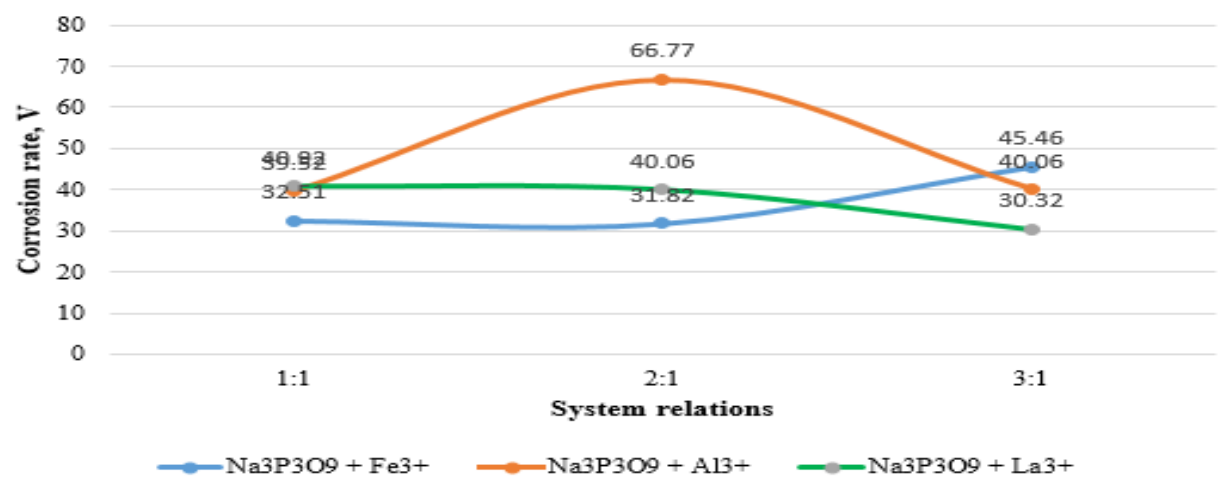

Fig. 6. Dependence of the corrosion rate on the nature of the modifier ion at a different ratio for sodium cyclotriphosphate.

As can be seen from Figure 6, as well as for diphosphates, cyclophosphates in the presence of iron ions with an increase in the concentration of phosphate are characterized by a decrease in the corrosion rate at a ratio of 2:1, and at a ratio of 3:1 it increases back. An inverse relationship is observed for a system with an aluminum modifier ion. In the system with the lanthanum ion, a gradual decrease in the rate of the corrosion process with increasing concentration is observed. As mentioned above, perhaps this is due to the formation of a complex compound, which reduces the corrosion process.

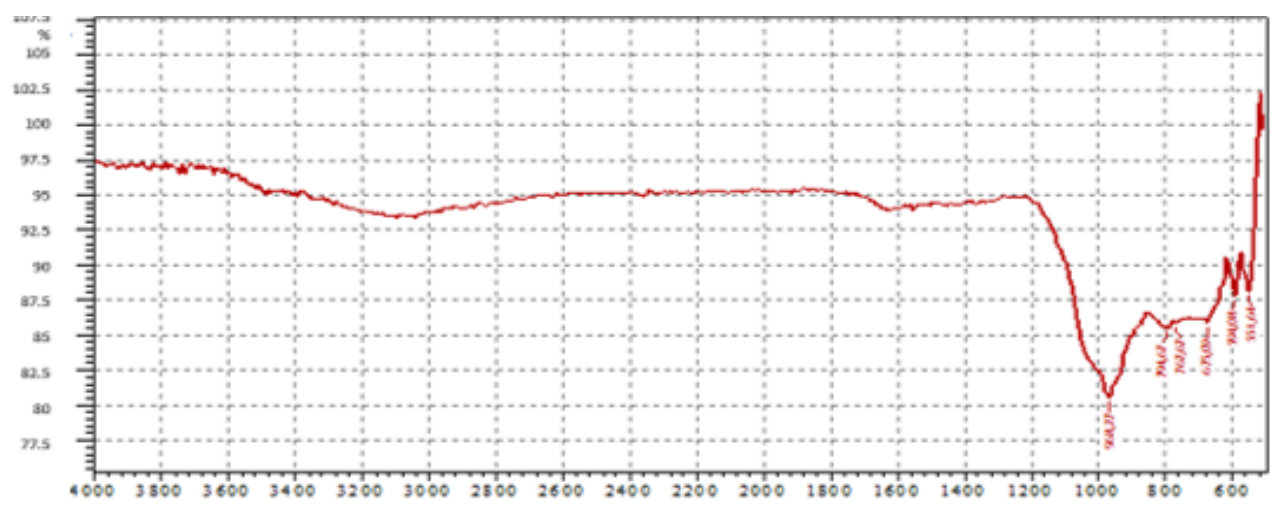

Fig. 7. Spectrogram of the corrosion deposition of the composition of sodium cyclotriphosphate with lanthanum in a ratio of $3: 1$.

Figure 7 shows a spectrogram of the corrosion deposition of the sodium cyclotriphosphate system with lanthanum in a ratio of 3:1. From literary sources it is known that for sodium hydrogen phosphate, the bands of $1225 \mathrm{~cm}^{-1}$ are characteristic for asymmetric $\vee(\mathrm{P}=\mathrm{O})$, and the band for 1294-1262 $\mathrm{cm}^{-1}$ - for the symmetric $\vee(\mathrm{P}=\mathrm{O})$ vibrations. The bands at $1168 \mathrm{~cm}^{-1}$ correspond to asymmetric $v$ (P-O-P), and the bands at $1162-1121 \mathrm{~cm}^{-1}$ correspond to the symmetric $v(\mathrm{P}-\mathrm{O}-\mathrm{P})$ vibrations. In the IR spectrum of 
the corrosion deposition, the peaks are not similar to the data from literature, which suggests that another compound was formed as a result, which forms a protective film.

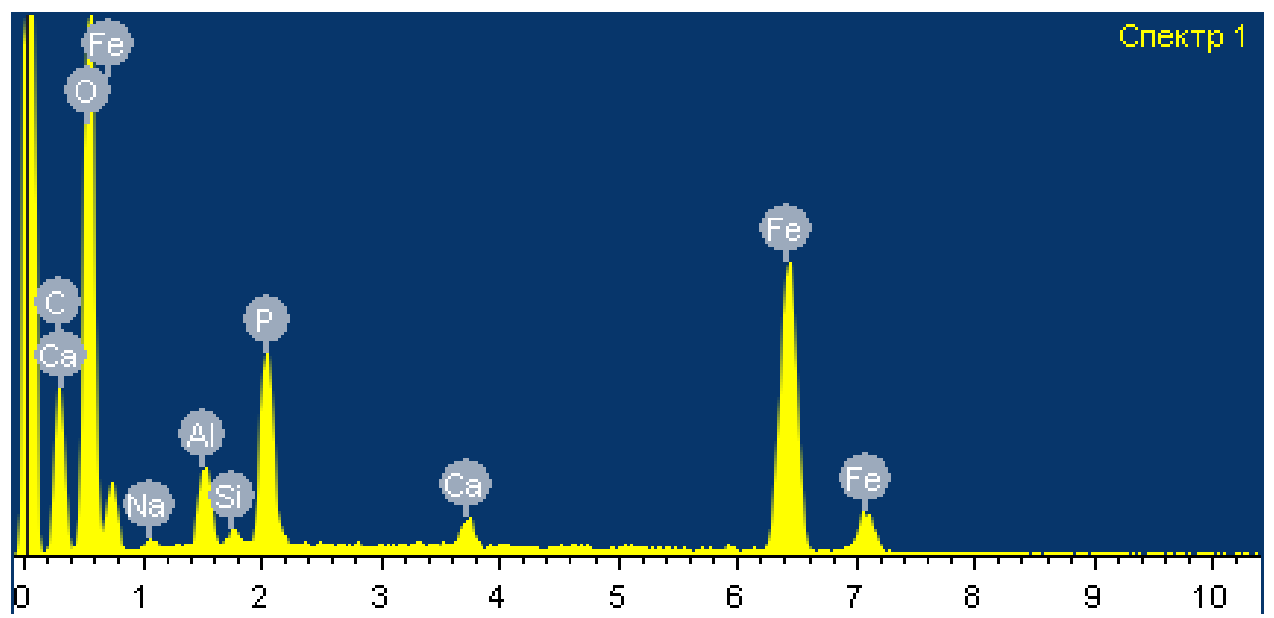

Fig. 8. Spectral scale of elements for corrosion deposition of the composition of sodium cyclotriphosphate with lanthanum in a ratio of 3:1.

Figure 8 presents an analysis of corrosion deposits on a scanning electron microscope and showed that oxygen $-36.97 \%$, iron $-28.68 \%$, phosphorus $-5.24 \%$ prevail in the corrosion deposits of this system. A small amount contains sodium, carbon.

Table 1. Basic thermodynamic indicators of multicomponent diphosphate and cyclophosphate systems.

\begin{tabular}{|c|c|c|c|c|c|}
\hline № & $\begin{array}{c}\text { Inhibitor }+\mathbf{L a}^{\mathbf{3}} \\
\text { in the ratio 1: } \mathbf{1}\end{array}$ & $\begin{array}{c}-\Delta \mathbf{G}^{\mathbf{0}} \mathbf{2 9 8 . 1 5}, \\
\mathbf{k J} / \mathbf{m o l}\end{array}$ & $\Delta \mathbf{H}, \mathbf{1 0}^{-\mathbf{5}} \mathbf{k J} / \mathbf{m o l}$ & $\Delta \mathbf{S}, \mathbf{1 0} \mathbf{0}^{-\mathbf{3}} \mathbf{k J} / \mathbf{m o l}$ & $\mathbf{l g K}$ \\
\hline 1 & $\mathrm{Na}_{2} \mathrm{H}_{2} \mathrm{P}_{2} \mathrm{O}_{7}$ & $21.25 * 10^{4}$ & -12.71 & 713.36 & 37.34 \\
\hline 2 & $\mathrm{Na}_{4} \mathrm{P}_{2} \mathrm{O}_{7}$ & $21.17 * 10^{4}$ & -1.51 & 750.56 & 37.28 \\
\hline 3 & $\mathrm{Na}_{3} \mathrm{P}_{3} \mathrm{O}_{9}$ & $21.77 * 10^{4}$ & -10.95 & 730.64 & 38.24 \\
\hline 4 & $\mathrm{Na}_{6} \mathrm{P}_{6} \mathrm{O}_{18}$ & $21.3 * 10^{4}$ & -4.71 & 714.91 & 37.42 \\
\hline
\end{tabular}

Table 1 presents the thermodynamic characteristics of diphosphate and cyclophosphate systems with ion modifiers. The highest Gibbs energy for diphosphate systems is characteristic of the sodium dihydrogen diphosphate system.

Diphosphate ions are metastable in an aqueous solution and their protective effect under the experimental conditions is somewhat low $[15,16]$. The mechanism of action of diphosphates is that, when the corrosion process proceeds in background media, according to literature data, they form relatively soluble complex compounds of the $\mathrm{Me}\left[\mathrm{Me}_{2}\left(\mathrm{P}_{2} \mathrm{O}_{7}\right)_{2}\right]$ type, which are stable in weakly acidic media $\mathrm{pH}=3-5$, which shifts the equilibrium of the corrosion process towards metal dissolution $[17,18]$.

$\Delta \mathrm{G}^{0}{ }_{298 \cdot 15}$ of the cyclotriphosphate system is $-21.77 * 10^{4}$. This confirms that this composition has the greatest inhibitory effect among cyclophosphate compositions. 


\section{Conclusion}

Based on the obtained experimental data, the following conclusions can be drawn:

1. An increase in the concentration of phosphate in most cases leads to a deceleration of the corrosion process and the $\mathrm{pH}$ is shifted to the slightly alkaline side in all the studied systems.

Diphosphate systems showed the lowest rates of corrosion rate and caused a shift in the $\mathrm{pH}$ to the acidic region, which is associated with the metastable state of the phosphates themselves [19,20].

2. Quantitative indicators of the effectiveness of modified di- and polyphosphate inhibitors are determined. The degree of protection in the $\mathrm{Na}_{3} \mathrm{P}_{3} \mathrm{O}_{9}+\mathrm{La}^{3+}$ system is $45.86 \%$ and the value of the corrosion resistance of the metal based on the value of the depth index of corrosion is 4 points (according to GOST 5272-90).

3. The experimental data were confirmed by thermodynamic calculations to determine the stability of the system in the presence of modified inhibitors. The highest value of Gibbs energy is also characteristic of the sodium tripolyphosphate system with an ion modifier lanthanum in a ratio of $3: 1\left(\Delta \mathrm{G}^{0}{ }_{298 \cdot 15}=-21.77 * 10^{4}\right)$.

36 compositions were studied and based on the experimental data obtained, it was established that the most practically significant protective effect was shown by $\mathrm{Na}_{3} \mathrm{P}_{3} \mathrm{O}_{9}$ systems with a concentration of $0.05 \mathrm{~mol} / \mathrm{dm}^{3}$ with $\mathrm{La}^{3+}$ and a concentration of $0.025 \mathrm{~mol} / \mathrm{dm}^{3}$ with $\mathrm{Fe}^{3+}$.

Currently, Kazakhstan corrosion inhibitors are inferior to international standards in technological and economic parameters. The main task of the coming years is the creation in Kazakhstan of our own effective reagents and the expansion of industrial production, and their introduction into the national economy. The production of our own corrosion inhibitors will reduce the dependence on the import of expensive imported supplies, which necessarily require additional analytical quality control at the places of consumption.

The correct and effective use of the country's natural resources will contribute to sustainable economic growth and better people's lives.

The regularities established during the work expand and supplement the existing ideas about the protective effect of compositions based on inorganic phosphate compounds. The results obtained significantly contribute to the creation of effective, environmentally friendly and economically feasible steel corrosion inhibitors based on Kazakh raw materials. The data obtained make a certain contribution to the scientific field of effective inhibitory protection of metals.

\section{References}

1. E. McCafferty, Introduction to Corrosion Science. Springer, New York, NY, (2010).

2. J. Vosta and J. Eliasek, Study on Corrosion Inhibition from Aspect of Quantum Chemistry, Corros. Sci., 11, 223 (1971)

3. N.P. ZHuk, The course of the theory of corrosion and metal protection (Moscow, Metallurgy, 1976)

4. G.G. Ulig, R.U. Revi, Corrosion and the fight against it. Introduction to Corrosion Science and Technology (Leningrad, Chemistry, 1989)

5. D.I. Bregman, Corrosion inhibitors (Moscow, Chemistry, 1966)

6. S.M. Reshetnikov, Inhibitors of acid corrosion of metals (Leningrad, Chemistry, 1986)

7. T.N. Vladimirskaya, M.L. Chepelevetsky, Studies of complexation in the systems $\mathrm{Fe}^{3+}-\mathrm{P}_{3} \mathrm{O}^{5-} 10$ $-\mathrm{H}_{2} \mathrm{O}$ and $\mathrm{Fe}^{3+}-\mathrm{P}_{2} \mathrm{O}^{4-}{ }_{7}-\mathrm{H}_{2} \mathrm{O}$ (In: Chemistry and technology of condensed phosphates, 1989) 
8. A.B. Niyazbekova, N.V. Akatyev, G.K. Sulekeshova, T.A. Shakirov, Chromatographic study of systems cyclotri-, cyclotetra- and cyclohexaphosphate with two and trivalent cations of $p$-and $d$-elements, Technology «Materials of the vi international research and practice conference», Materials of the VI International research and practice conference Munich, Germany, pp. 186194 (2013)

9. G.S. Kuanysheva, G.R. Makasheva Г.P., G. Kamalova, A.B. Niyazbekova, Complexation of salts of some d-elements with diphosphate anion, Bulletin of Karaganda State University, v. 1 (13), pp. 71-73 (1999).

10. D.E.C. Corbridje, The structural of chemistry of phosphates, Bull. soc. fr. miner. et. cristallog. v. 94, pp. 271-299 (1971)

11. U.F. Zhdanov, Chemistry and technology of polyphosphates (Moscow, Chemistry, 1979)

12. A.V. Laserov, L.V. Kubasova, N.N. Chudinova, Income and research and alkali metal phosphates, v. 18, pp. 127-131 (1982)

13. A. Durif, The development of cyclophosphate crystal chemistry, Solid State Sciences, v. 7, pp. 760-766 (2005)

14. R. Pearson, Hard and soft acids and grounds, Successes of chemistry, v. 15, pp. 1259-1271 (1974)

15. I.B. Tananaev, A.B. Lavrov, N.N. Chudinova, The study of phosphates, v. 33, pp. 2041-2048 (1988)

16. N. Narayana, M. Rao, R. Gómez-García, A. Kornberg, Inorganic Polyphosphate: Essential for Growth and Survival, An- nual Review of Biochemistry, v. 78, pp. 605-647 (2009)

17. M.Kh. Karapetov, S.I. Drakin, General and inorganic chemistry (Moscow, Chemistry, 1981)

18. Kura Genichiro, Hydrolysis reaction of inorganic cyclophosphates at various acid strengths, Polyhedron, v. 6, pp. 531-533 (1987)

19. G.S. Kuanysheva, S.S. Dauletkalieva, Experimental and quantum-chemical studies of complexes of iron (III) with diphosphate in solution, // Mater. XXV Int. Chugaev Conf. in coordination chemistry, pp. 337-338 (2011)

20. A. Niazbekova, N. Akatyev, M. Mukasheva, A. Rakhova, Quantum- chemical calculations of electronic structure of polyphosphate complexes of manganese, cobalt, copper and zinc, Materials of the international research and practice conference «European Science and Technology», Wiesbaden: Germany, pp. $82-85$ (2012) 